\section{NIV IN INFANTS AND CHILDREN WITH ACUTE RESPIRATORY FAILURE - PRACTICAL ISSUES}

\section{B. Schmitt}

\section{University Children's Hospital, University Medicine Mainz, Mainz, Germany}

Non - invasive ventilation (NIV) is defined as the use of a mask or interface to provide ventilatory support.

Avoiding endotracheal intubation and reducing the rate of complications and mortality secondary to intubation is the most important advantage.

Inadults noninvasive positive pressure ventilation (NIPPV) is used as a first line intervention in acute respiratory failure (ARF). Many trials have proven the beneficial effects of NIPPV in adult patients with chronical deseases.

In pediatric patients with acute respiratory failure NIPPV is still not as well established. There is still a lack of suitable interfaces for pediatric patients especially in small infants.

Resting upon more than ten years experience in treatment of infants and children with NIPPV in ARF I will focus on practical issues and pitfalls concerning noninvasive ventilation in these group of patients.

The presentation covers both the published experience and practical aspects of noninvasive ventilation in pediatric patients with ARF.

Apart from mentioning different interfaces and ventilators it is more important to show how to deal with the interfaces and the ventilators.

We have to do with questions concerning e.g. skin damage by ventilation interfaces,

ineffective humification, air leaks by gastric tubes, noncompliant patients and how to proceed whith critical ill children in ARF.

The keypoint of NIPPV in infants and children is the interdisciplinary collaboration

of doctors and caregivers.

Improvement of NIV in pediatrics is a question of continuous training and education of your team in the ward.

\section{ACUTE PULMONARY LESION CAUSED BY MECHANICAL VENTIALTION}

\section{H.A. Urbina-Medina}

Pediatric Critical Care, Universidad Central de Venezuela, Hospital de Niños JM de los Ríos,

Caraca, Venezuela

Introduction: mechanical ventilation is a necessary therapy in critical pathologies with respiratory failure, producing acute pulmonary injury.

Objectives: To identify an acute pulmonary injury by bronchioalveolar lavage cytology and determine causal relations with the Fraction of inspired Oxygen (FiO2), the pressure of the airways, positive endexpiratory pressure (PEEP), oxygen saturation and exposure time.

Methods: Longitudinal, prospective clinical investigation from January to July 2007, which included 51 patients that received mechanical ventilation. 153 bronchioalveolar lavage citologies were obtained and processed.

Results: Several alterations in citologies were observed at 12, 72 and 144 hours after mechanical ventilation was started, which shows a simple and meaningful statistic association with fraction of given oxygen at 12 and 74 hours, PEEP at 12 hours, Pressure in air ways and and oxygen.

Discussion: It becomes evident that alterations produced by mechanical ventilation are connected with FiO2, oxygen saturation, absence or low PEEP and elevated airway pressure.

Conclusion: Alterations in citologies of the bronchioalveolar epithelium present themselves in the presence of FiO2 and low PEEP.

Key words: Acute pulmonary injury. Mechanical ventilation. Bronchioalveolar lavage. 